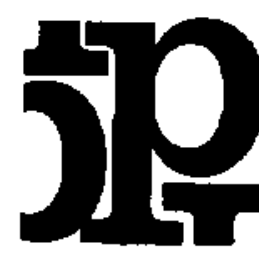

ARTICULOS

ORIGHALES

\title{
Diabetes infantil
}

DRS. : IRIS MELLA G. *. MANUEL GARCLA DE lOS RIOS A. * GLORIA LOPEZ $s . " y$ ERICH SCHILLING F. ${ }^{\text {* }}$,

INTRODUCCIÓN. En la clasificación actual de la Diabetes Mellitus (1), se considera Diabetes Juvenil aquella que se inicia antes de los 30 años de edad. Esta nueva denominación, más amplia, incluye al grupo anteriormente conocido como Diabetes Infantil, que clásicamente se limitaba a los pacientes cuya enfermedad comenzaba antes de los 15 años.

Si bien hay muchas razones para que esta forma de clasificación sea aceptada universalmente, nosotros creemos que el concepto de Diabetes Infantil debe mantenerse en cualquier sistema de clasificación con cierta independencia, debido fundamentalmente a que corresponde a un período especial de crecimiento, pubertad y desarrollo del ser humano.

La diabetes del viño es una enfermedad infrecuente, 1 por cada 2.500 , según cifras extranjeras (2), no existiendo lamentablemente datos nacionales. A pesar de esta baja incidencia, por tratarse de una afección crónica, en los servicios especializados se logra reunit a través de los años una buena experiencia en el diagnóstico y manejo de estos pacientes, que siempre constituyen un problema difícil para los médicos generales.

En el Departamento de Nutrición y Diabetes del Hospital San Juan de Dios, hemos reunido un grupo importante de casos, lo que nos ha estimulado a realizar este análisis, que creemos serâ de utilidad para conocer y divulgar la realidad de la Diabetes Infantil en nuestro medio.

Material y método. Hemos tenido la oportunidad de estudiar y tratar 110 diabéticos infantiles; de este número han fallecido 12 pacientes y

\footnotetext{
"Departamento de Mcdicina, Area Occidente, Universidad de Chile. Hospital San Juan de Dios.

* Departamento de Pediaitía, Area Occidente, Universidad de Chile. Hospita] San Juan de Dios.
}

35 tuvieron una observación menor a $\operatorname{los} 2$ años, por lo cual no han sido considerados en este trabajo.

Nuestro material está constituido por un grupo seleccionado de 63 casos, 29 hombres y 34 mujeres, controlados por nosotros bajo iguales condiciones de estudio y que se encuentran en la actualidad concurriendo al Departamento por un período superior a los 2 años. Todo el material, excepto 1 caso, es de tipo inestable, característica inherente a la diabetes infantil. Todos han sido sometidos a controles metabólicos similares, exámenes complementarios rutinarios, fondo de ojo periódico, abreu y radiografía de mano para determinación de edad ósea. Además, se realizó en este grupo Encuesta social y Dietética para determinar el grado de cumplimiento de nuestras indicaciones. Los pacientes recibieron indicación dietética calculada, con instrucción personal por nutricionistas, como hemos publicado anteriormente (3).

La distribución de nuestro material por edad de comienzo de la enfermedad y sexo aparecen en la tabla $N o$ 1, destacándose una leve preponderancia del sexo femenino, siendo para ambos

TAB L A No 1

DIABETES INFANTIL EDAD DE COMIENZO $Y$ SEXO

\begin{tabular}{|c|c|c|c|c|}
\hline \multirow{2}{*}{$\begin{array}{l}\text { Edad } \\
\text { años }\end{array}$} & \multicolumn{2}{|c|}{ Masculino } & \multicolumn{2}{|c|}{ Femenino } \\
\hline & $N^{o}$ & $\%$ & $N^{o}$ & $\%$ \\
\hline $0-5$ & 9 & 31,0 & 4 & 11,7 \\
\hline $6-10$ & 11 & 38,0 & 20 & 58,8 \\
\hline $11-15$ & 9 & 31,0 & 10 & 29,4 \\
\hline Total & 29 & $100 \%$ & 34 & $100 \%$ \\
\hline
\end{tabular}


grupos más frecuente la iniciación entre los 6 y 10 años de edad. lo que es mús marcado para las mujeres. El promedio de iniciación para ambos sexos es de 8,1 años, con un rango de 1,5 a 15 años.

Al realizar el trabajo 42 diabéticos $(66,6 \%)$ se encuentran en edades comprendidas entre los 11 y 20 años, lo que da al grupo caracteristicas especiales, por ser la adolescencia un período singularmente difícil en el manejo de la enfermedad debido at su inestabilidad psíquica y metabólica.

Debcmos anotar que el grupo de pacientes estudiados tiente on so gran mayoria unal reliliva antigüedad do l:1 diabetes, como se presentu in la tabla No 2. ya yut sólo bا $35 \%$ tienc menos de 5 años de enterniedad y un 32\% está sobre los 10 años. Las cifras son semejantes para ambos sexos con un promedio de 8.3 años de evolución.

\section{IABI A NY 2}

DIABETES INFANTIL AÑOS DE EVOLUCION $Y$ SEXO

\begin{tabular}{lcccc}
\hline $\begin{array}{c}\text { Años } \\
\text { Diabetes }\end{array}$ & Masculino Femenino & No Totat & $\%$ \\
\hline $0 \cdot 5$ & 10 & 12 & 22 & 35 \\
$6 \cdot 10$ & 10 & 11 & 21 & 33 \\
$11-15$ & 7 & 10 & 17 & 27 \\
$16-20$ & 2 & 1 & 3 & 5 \\
\hdashline Total & 29 & 34 & 63 & 100
\end{tabular}

A continuación analizaremos una serie de características clínicas de cste material, aparición de complicaciones, desarrollo pondo estatural y escolaridad.

Resultados. 1.- Antecedentes herediturios. De la investigación anamnéstica acuciosa logramos obtener el antecedente de diabetes familiar en el $45 \%$ de los casos, con una leve mayor frecuencia en el grupo de los hombres ( $55 \%$ ) con respecto a las mujeres $(35,8 \%)$. No hubo diferencia en la distribución hereditaria de línea materna o paterna.

2.- Forma de comienzo. En 24 casos (38\%) el diagnóstico se hizo a raíz de un coma diabético y un $8 \%$ en una acidosis importante que requirió hospitalización, pero sin Ilegar al coma. En el resto de los casos el diagnóstico fue más oportuno, en la etapa de los síntomas clásicos de descompensación. No hubo diferencias significativas entre ambos sexos. Destacamos el comienzo rápido e incluso súbito del cuadro, hecho común en casi todos los pacientes; ya que sólo en 3 casos se consignó una iniciación gradual de la sintomatología.
Al relacionar la edad de comienzo de la diabetes y su forma de iniciación, encontramos que en el grupo menor de 5 años más del $50 \%$ iniciaron el cuadro en coma.

3.- Remisión. Consiste en una disminución substancial del requerimiento insulínico del niño Jespués del periodo inicial de tratamiento. Del to. tal de nuestro material, 9 casos $(14,3 \%)$ hicieron una remisión que se presentó entre 2 y 5 meses después de iniciada la diabetes. La duración totil oxciló entre 2 meses y 2 años como máxinu. Fa 4 tasos fuc súlo parcial, disminuyendo of $\mathrm{rt}^{2}$ yutrimiento de insulina a dusis variables entre 8 y 10 Unidades diacias y en 5 cusos fue total; de éslos, + tueron tratados con Fenformina en dosis de 50-75 mgrs. diarios durante todo su período de remisión.. No so observaron diferencias en reJación al sexo.

4.- Tratamiane insulintico: a) Dosis inicial. Se analizo on un grupo seleccionado de 35 pacientes que fueron controlados por nosotros desde el comienzo de su enfermedad, en etlos pudimos relacionar en forma exacta el requerimiento inicial de insulina con el peso del niño. En 13 casos (37\%) este fuc de $1 / 2$ unidad por $\mathrm{Kg}$. de peso, en $16(45 \%)$ entre $1 / 2$ y 1 unidad por $K g$. de peso y finalmente en 6 cases $(18 \%)$ de 1 unidad por Kg. de peso.

b) Esquemas insulínicos. Al fijar las características de nuestro material, dijimos que todos tenían mấs de 2 años de diabetes controlados en el servicio $y$ es ampliamente conocido el hecho que justamente en los dos primeros años de enfermedad la diabetes evoluciona en forma algo más estable y por ende, susceptible de un tratamiento insulínico más simple.

Del análisis del grupo se desprende que en 38 casos $(60,3 \%)$ se usaron mezclas de Insulina lenta y cristalina en dosis variable, en 1 inyección diaria; 17 casos $(25,4 \%)$ recibieron una dosis de Insulina Lenta. En 5 pacientes se usaron 2 dosis de Insulina Lenta y en 2 casos fue necesario usar Insulina corriente fraccionada, por su mayor inestabilidad. Finalmente, en un caso realmente estable desde el comienzo, fue posible controlarlo con drogas orales.

c) Dosis actuales. El requerimiento inicial de insulina se eleva a medida que el nin̆o crece, sube de peso y aumenta la antigüedad de la diabetes, llegando a su máximo entre los 6 y 10 años de la enfermedad para posteriormente decrecer.

En la tabla No 3 aparece relacionada la edad de los niños, los años de diabetes y el promedio de insulina que usan en la actualidad en cada grupo. Se observa con claridad lo señalado más arriba, destacándose que el grupo de mayor requerimiento insulínico es el comprendido entre 16 y 20 
años de edad y 6 a 10 años de diabetes, que alcanza a 81 unidades diarias como pronedio.

En la diabetes infantil es muy difícil evaluar el resultado a través del control metabólico, dada su gran inestabilidad y descompensaciones frecuentes por cuadros infecciosos agregados. Así y todo, nosotros clasificamos nuestros resultados en bueno, regular y mılo, de acuerdo a los standards babituales en uso en el Departamento. Para este fin calculamos promedios de glicemias y glucosurias en el curso de los años, con cierta flexibilidad para casos de descompensación ocasional por factores intercurrentes. Con estas salvedades, tenemos 12 casos considerados buenos $(19 \%), 21$ regulares $(33,3 \%)$ y 30 malos $(47,6 \%)$.

A todo el grupo se le hizo una encuesta de alimentación por las nutricionistas, según método de tendencia de consumo, observándose que aque-

TABLA No 3

DIABETES INFANTIL EDAD, ANOOS DE DIABETES $Y$ REQUERIMIENTO CONTROL INSULINA

\begin{tabular}{rcccc}
\hline $\begin{array}{c}\text { Edad } \\
\text { años }\end{array}$ & $0-5$ & $\begin{array}{c}\text { Años de Diabeies } \\
6-10\end{array}$ & $11-15$ & $16 y+$ \\
\hline $0-5$ & 14 & & & \\
$6-10$ & 38 & 49 & & \\
$11-15$ & 58 & 63 & 42 & \\
$16-20$ & 53 & 81 & 67 & \\
$21 y+$ & & 74 & 56 & 50 \\
\hline
\end{tabular}

Hos con control metabólico bueno coincidían en general, con un cumplimiento adecuado de Ja cuota de Hidratos de Carbono o bien con una reducción mayor, realizada por propia iniciativa; mientras que los de mal control, correspondían a aaquéllos con excesiva ingestión de Hidratos de Carbono o con ingesta muy irregular. En dos ninos muy lábiles no existía ninguna relación entre el cumplimiento de la dieta y los controles metabólicos. Además intentamos relacionar el grado de control metabólico ya señalado, con la cooperación de los padres al cumplimiento de las indicaciones y al interés demostrado en el cuidado del niño. Encontramos una relación directa entre anbos hechos, salvo los 2 casos extremadamente lábiles ya anotados.

Finalmente, la Asistente Social hizo un acucioso estudio de tipo socio-económico pensándose que podría existir alguna relación entre mejores condiciones de vida y control; sin embargo, no encontramos la relación señalada.

5.- Lipodistrofia. En nuestro material sólo hemos observado la variedad atrófica, nunca la forma hipertrófica del tejido celular subcutáneo en los puntos de colocación de insulina. No he- mos encontrado relación entre lipodistrofia y dosis o tipo de insulina usada. Observamos $15 \mathrm{ca}$ sos entre las niñas (44\%) y sólo $6(20 \%)$ en los ıй̄os, sicndo 2 de ellos muy leves. En Jas mujeres, 5 de los 15 casos, se recuperaron totalmente al cambiac definitivamente el sitio de la inyección.

6.-Desarrollo óseo. Estudiamos la edad ósea mediante la técnica habitual de radiografía de mano izquierda en 50 nin̄os, que corresponden a aquélos menores de 21 años. Se destaca, como aparece en la tabla No 4 , que el $46 \%$ presenta una edad ósea disminuida, en su mayoría en 1 a 3 años con un caso extremo de 7 años; este hecho es más frecuente en el sexo masculino que en el femenino (14 y 9 respectivamente). Edad ósea normal en el $40 \%$ y aumentada en el $14 \%$, correspondiendo 6 casos a las mujeres y 1 a los hombres.

TABLA No 4

DIABETES INFANTIL EDAD OSEA Y SEXO EN $5 O$ DIABETICOS INFANTILES MENORES DE 21 $A N T O S$

\begin{tabular}{|c|c|c|c|c|}
\hline $\begin{array}{l}\text { Edud } \\
\text { óseu }\end{array}$ & $\begin{array}{c}S_{e} \\
\text { Femenino }\end{array}$ & $\begin{array}{l}x o \\
\text { Masculino }\end{array}$ & Total & $\%$ \\
\hline Normal & 13 & 7 & 20 & 40,0 \\
\hline Disminuida & 9 & 14 & 23 & 46,0 \\
\hline Aumentadat & 6 & 1 & 7 & 14,0 \\
\hline Total & 28 & 22 & 50 & 100,0 \\
\hline
\end{tabular}

7.- Desarrollo pondo estatural. Nuestros resultados los analizamos en peso y talla en forma separada para hombres y mujeres hasta los 18 años, por considerar que a esa edad se ha completado el desarrollo pondo estatural.

En la figura No 1 aparece la relación de edad y peso para el grupo de los hombres, proyectada

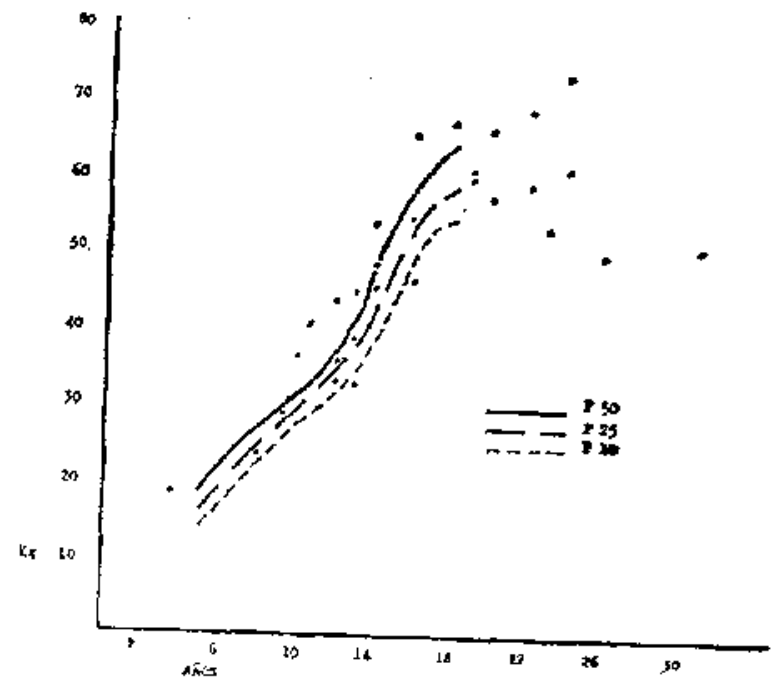

Figura No 1.- Diabetes Infantil. Edad g peso. Sexo masculino. 


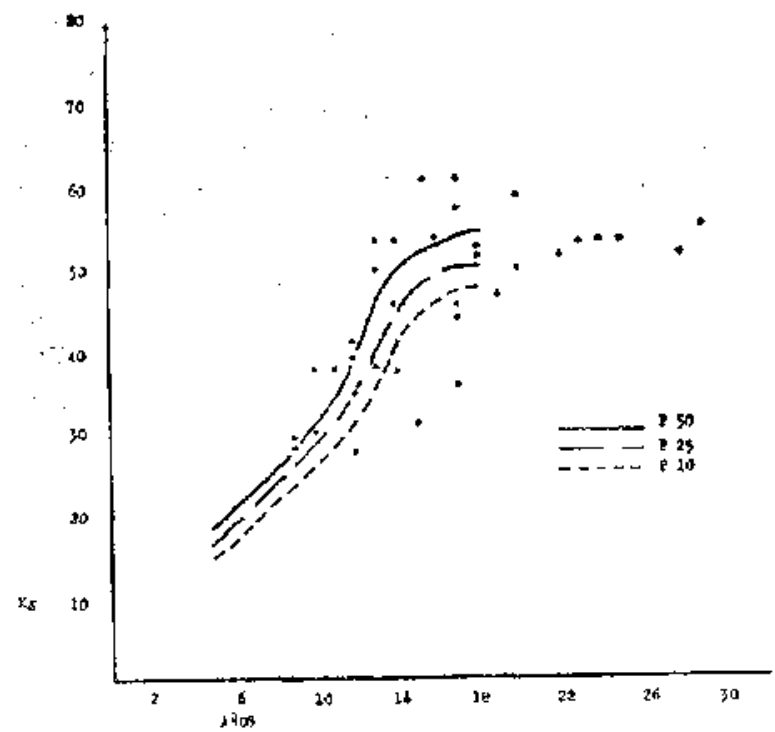

Figura N: 2.- Diabetes infanti]. Edad y neso. Sexo masculino.

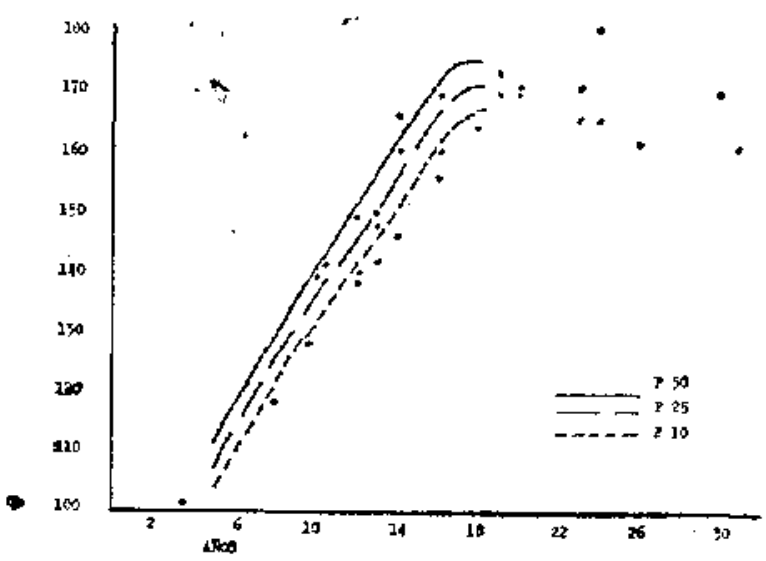

Figura Nọ 3,- Diabetes Iníantif. Echad y talla. Sexo masculino.

sobre las Tablas de Iowa, observandose que de 18 niños hasta los 18 años de edad, 8 están sobre el percentil 50,6 entre el 50 y 25,2 entre el 25 y 10 y sólo 2 bajo el percentil 10 .

En la figura $\mathrm{N}^{\circ} 2$ se relacionan las mismas variables para las mujeres, destacándose que de 25 niñias, 11 están sobre el percentil 50,6 entre el 50 y 25,2 entre el 25 y 10 y finalmente 6 bajo el percentil 10.

El análisis de la talla, siempre en los menores de 18 años, nos da en los hombres, 1 solo sobre el percentil 50, 6 entre el 50 y 25,2 entre el 25 y 10 y 9 bajo el percentil 10 , como se aprecia en la figura No 3.

Finalmente en la figura $N$ ? 4 se ve la taila de las niñas, de las cuales 1 está sobre el percentil 50,3 entre el 50 y 25,7 entre el 25 y 10 y 14 bajo el percentil 10 .

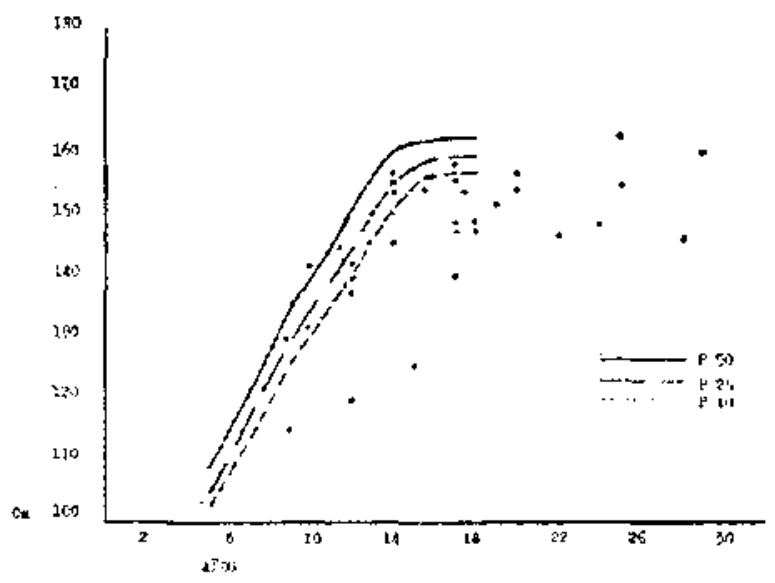

Figura Va 4-- Diahetck infantil. Elad y talla. Scxu femenino.

En relación a los diabéticos infantiles actualmente mayores de 18 años y que podemos considerar adultos en su desarrollo, encontramos el mismo déficit de peso y talla, siempre más marcado en el sexo femenino. Ahora, si relacionamos estas variables, usando la Tabla de peso aceptable de Valiente y Taucher (4), vemos que 6 hombres de un total de 11 y 3 mujeres de 9 , están por debajo del peso mínimo aceptable.

8.- Desarrollo sexual. a) Femenino. Estudianos los caracteres sexuales femeninos y la aparición de menarquia en 26 casos. De éstas, 25 han presentado su menarquia con un rango que varió entre 11 y 16 años, con un promedio de 13,3 años; el único caso de amenorrea primaria corresponde a una nin̆a con un déficit pondo-estatural importante, que está siendo estudiada en endocrinología por una probable aberración cromosómica. Continúan con menstruaciones normales 20 casos, los otros 5 presentan diversos grados de alteraciones del ritmo menstrual. Los caracteres sexuales secundarios se presentaron en forma normal.

Una paciente ha tenido 4 embarazos, con 2 nirios vivos y 2 muertos por parto prematuro a los 6 meses por insuficiencia placentaria, pese al tratamiento hormonal sustitivo.

b) Masculino. Los caracteres sexuales secundarios se estudiaron en niños mayores de 14 años, siendo en todos normales. No hemos observado problemas de líbido. Un joven de 31 años de edad y 17 de diabetes, presenta una impotencia por neuropatía diabética que ha sido irrecuperable.

9.- Escolaridad. Con el objeto de analizar el grado de instrucción y por ser tan amplio el rango de edad, hemos agrupado este material en menores y mayores de 18 años, como se observa en la tabla No 5.40 niños corresponden a menores de 18 años en edad escolar, en 25 la escolaridad es normal (cursan básica o enseñanza media en 
T А B I. ^ No 5

\begin{tabular}{|c|c|c|}
\hline Escolatidad & \multicolumn{2}{|c|}{$E d a d$} \\
\hline \multicolumn{3}{|l|}{ Normales } \\
\hline Fscolares & 25 & - \\
\hline Universitarios & - & 4 \\
\hline I icenciados &.-- & 4 \\
\hline Atrusados & 1.3 & 7 \\
\hline Abandonan sus estudios & 2 & 7 \\
\hline To & 40 & 22 \\
\hline
\end{tabular}

relación a la edad), 13 van atrasados y 2 abandonaron sus estudios.

De los 22 casos mayores de 18 años, 4 son licenciados y 4 tienen estudios universitarius. Analizando el grupo en total se observa que el $53,2 \%$ cursan básica, enseñanza media o universitaria en el grado correspondiente a su cdad cronológica y $32,2 \%$ van atrasados en relación a su edad.

10.- Complicaciones agudas. a) Keto-Acidosis diabética. En todas nuestras publicaciones (5) hemos seguido el concepto químico para definir el coma diabético, considerando como tal, aquel paciente que presenta un $\mathrm{CO} 2$ inferior a $9 \mathrm{mEq} \%$, cualquiera que sea su condición neurológica en ese momento; este concepto, discutible para algunos, tiene para nosotros un gran valor terapéutico y pronóstico.

De Ios 63 casos estudiados, 34 han ingresado al Departamento u otros servicios de urgencia por Ketoacidosis diabética. Como se puede apreciar en la tabla $N^{\circ} 6$, se han presentado 11 comas $(17,5 \%)$ y 23 acidosis $(36,5 \%)$.

Estas complicaciones no se observaron en ningún momento de la evolución en 29 pacientes $(46 \%)$. Además se consigna en el cuadro algunos pacientes que han repetido alguna forma de Ketoacidosis, de tal modo que los 34 enfermos han tenido en total 60 episodios de Ketoacidosis.

En la mayoría de los casos hemos podido pre-

TABLA No 6

DIABETES INFANTIL KETO-ACIDOSIS DIABETICA

\begin{tabular}{lccll} 
& Masctino & Femenino & Toial & $\%$ \\
\hline Coma diabético & 3 & 8 & $11^{\text {* }}$ & 17,5 \\
Acidosis & 12 & 11 & $23^{\text {\%* }}$ & 36,5 \\
Sin Ketoacidosis & 14 & 15 & 29 & 46,0 \\
\hline Total & 29 & 34 & 63 & 100,0 \\
\hline
\end{tabular}

4 casos repitieron el coma 2 o más veces.

$* \quad 10$ casos repitieron la acidosis 2 o más veces. cisar el factor desencadenante de esta complicación. La causa más frecuente es la infracción dietética con el $41,6 \%$, luego suspensiones de Insulina $26,6 \%$ infecciones diversas $23,3 \%$ y no fue posible precisar la causa en el $8,3 \%$ de los casos.

b) Shock hipoglicémico. Hemos consignado sólo Ja hipoglicemia con pérdida de conciencia, dado que la reacción hipoglicémica es inherente al tratamiento insulínico y se presenta habitualmente en todos los casos. El 37,9\% de los niños $y$ el $26,4 \%$ de las niñas hicieron shock hipoglicémico.

c) Infecciones. Para el análisis de esta complicación no hemos considerado las infecciones respiratorias altas de tipo banal, ni las infectocontagiosas de la infancia por no tener relación con la enfermedad basal. Encontramos 31 pacientes de los 63 estudiados, que en conjunto presentaron 42 infecciones diversas, debido a que algunos hicieron la nisma u otra localización infecciosa a través de los años.

En la tabla No 7 se puede observar la relación existente entre el grado de control metabólico y diversas infecciones, para lo cual las separamos en piel y celular, urinarias, neumopatía, TBC y otras. No hubo diferencias por sexo. Llama la atención la mayor frecuencia de las infecciones de la piel y celular $(71,4 \%)$, seguidas de las urinarias $(14,2 \%)$. Debe destacarse que las infecciones urinarias se presentaron en pacientes con mal control metabólico, hecho también observado por nosotros en adultos. Finalmente queremos hacer notar que del total de infecciones, el $11,9 \%$ corresponden a casos con buen control metabólico, $23,8 \%$ a control regular y $64,2 \%$ a mal control.

11.- Complicaciones degenerativas. a) Retinopatía diabética. Presentan esta complicación 15 pacientes (23\%), 10 iniciales y 5 moderados. Su evolución oftalmológica ha sido apreciada en la sigutente forma: 5 casos regresivos, 2 estabilizados, 4 progresivos y 4 en etapa muy inicial, que no han sido controlados aún. Llamamos evolución

TABLA No 7

DIABETES INFANTIL LOCALIZACIONES INFECCIOSAS Y CONTROL METABOLJCO

\begin{tabular}{|c|c|c|c|c|}
\hline Localización & Bueno & Regular & Malo & Total \\
\hline $\begin{array}{l}\text { Piel y } \\
\text { celular }\end{array}$ & 3 & 9 & 18 & 30 \\
\hline Urinarias & & & 6 & 6 \\
\hline $\begin{array}{l}\text { Neumopatía } \\
\text { aguda }\end{array}$ & 1 & 1 & & 2 \\
\hline ТВC & 1 & & 1 & 2 \\
\hline Otros & & & 2 & 2 \\
\hline Total & 5 & 10 & 27 & 42 \\
\hline
\end{tabular}


Iegresiva aquella que habiendo presentado algún tjpo de lesión, ésta ha disminuido su intensidad o desaparecido. De los casos progresivos, 2 fueron sometidos recientemente a fotocoagulación por su mayor rapidez de evolución; el resultado final se podrá apreciar con el tiempo.

Clásicamente se plantea la relación existente entre la aparición de retinopatía con la antigüedad de la diabetes y grado de control metabólico; hechos que hemos tratado de demostrar, pese al número bajo de casos en la tabla No 8 . De él se desprende, que en el grupo con menos de 5 años de enfermedad existe 1 sola retinopatía, que coincide con el peor control. A medida que avanzan los años de enfermedad aparecen, aunque con baja frecuencia lesiones retinales con buen control, 1 en cada quinquenio, sienco siempre de mayor frecuencia esta complicación en los grupos regulares y malos.

$$
\begin{array}{lll}
\text { T A B L A } & \text { No } 8
\end{array}
$$

DIABETES INFANTIL RETINOPATIA, ANTIGUEDAD DE LA DIABETES Y GRADO DE CONTROL

\begin{tabular}{lcccc}
\hline Años diabetes & Bueno & Reguiar & Malo & Total \\
\hline $0-5$ & & & 1 & 1 \\
$6-10$ & 1 & 2 & 4 & 7 \\
$11-15$ & 1 & 1 & 5 & 7 \\
\hline T o t a 1 & 2 & 3 & 10 & 15 \\
\hline
\end{tabular}

* Tratamiento con droga oral.

Si relacionamos sólo presencia de retinopatía y grado de control, vemos que el $16,6 \%$ de los casos buenos presentan lesiones, frente al $33,3 \%$ de Jos considerados malos.

b) Albuminuria. Hemos encontrado la presencia de albúmina en la orina en 6 pacientes, $4 \mathrm{mu}-$ jeres y 2 hombres; en uno de cada caso coincidente con lesión de la retina, lo que nos permite en estos casos formular el diagnóstico de nefropatía diabética. Si relacionamos este halfazgo con el control metabólico, vemos que 4 corresponden a control malo y 2 buenos, siendo éstos con 10 y 15 años de evolución de la enfermedad.

c) Neuropatía diabética. Se ha tormulado cste diagnóstico en 8 casos, 4 para cada sexo, siendo más frecuente después de 6 años de enfermedad, sin relación con el grado de control. Sin embargo, la polineuropatía más intensa y dramática que tuvimos oportunidad de ver, correspondió a una niña diabética de tipo algo más estable, que había suspendido el tratamiento insulínico durante 2 años y que recibimos en un grave estado de desnutrición; se recuperó después de varios meses de tratamiento y una vez solucionado su problema nutritivo.
Comentakios. Lal distribución de nuestro miterial por sexo con una leve mayor frecuencia entre las mujeres, corresponde exactamente a la dc autores extranjeros (6-7) con la excepción de Knowles (8). FI antecedente hereditario de diabetes que nosotros encontramos cn el $45 \%$, es muy scmejante al de Oakley (7) que da el $40 \%$ en una serie de 516 casos.

La iniciaciôn en coma diabético, se presenta entre el $15 \%$ a $20 \%$ de los casos según revisiones bibliográficas (6-7-9), lo que debe considerarse un diagnóstico más oportuno frente a la nuestra del $38 \%$. En este valor tan alto incide fundamentalmente la demora en la consulta por parte de los pacientes, y entre los médicos, el no pensar oportunamente en el diagnóstico de diabetes infantil frente a los síntomas clásicos de descompensación, lo que se observa especialmente en el grupo bajo los 5 años de edad en que más del $50 \%$ inicia la enfermedad en coma.

Respecto a la etapa de remisión, es difícil comparar resultados porque los diferentes autores no fijan en forma clara los límites de disminución del requerimiento insulínjeo. La cifra de $30 \%$ de la Dra. White (6), que es el doble de la nuestra $(14,3 \%)$ y la más alta citada, la atribuye al exacto cumplimiento de las indicaciones terapéuticas desde el comienzo de la enfermedad.

En el tratamiento insulínico las dosis iniciales oscilaron entre $1 / 2$ y 1 unidad por $\mathrm{Kg}$. de peso $(82 \%)$, superiores a las de White que corresponden a $1 / 2$ unidad $x \mathrm{Kg}$. Otros autores relacionan estas dosis con la edad, to que nos parece poco práctico como norma general.

Es unánimemente aceptado que en la adolescencia, los diabéticos deberían recibir dos o más dosis de insulina para un adecuado control metabólico. Sin embargo, por razones socio económicas, sólo 7 casos de nuestro material se tratan en esta forma, en circunstancias que el $66 \%$ de los sujetos tiene edades que varían entre 11 y 20 años.

En relación a la evaluación del resultado metabólico, la literatura no proporciona datos, lo que confirma nuestra aseveración de la dificultad de medir estos parámetros que, sin embargo, pese a su poca cxactitud tienen algún valor pronóstico en relación a complicaciones crónicas đegenerativas.

La lipodistrofia, que en nuestro grupo se presenta en el $44 \%$ de las mujeres y sólo en el $20 \%$ de los hombres, para Knowles es del $20 \%$, igual para ambos sexos. White, (6) al igual que nosotros, la encuentra en casi todos los casos, sin relación con el tipo de insulina usada.

Nuestro estudio de edad ósea en este grupo parece original, al no tener datos concretos comparables en la literatura revisada. En este material llama la atención que el $46 \%$ tenga una edad 
ósea disminuida entre 1 y 3 años en relación a lo normal.

Se acepta unánimemente que los niños chilenos eutróficos son comparables en desarrollo a los de la curva de Iowa, de tal modo que nuestros diabéticos aparecen como francamente comprometidos en su crecimiento.

Del análisis pondo estatural de los menores đe 18 años, se destaca el mayor deterioro de la talla frente a los pesos, siendo impactante este hecho en las mujeres. Es así, como de 25 niñas, 21 están bajo el percentil $25 \mathrm{y}$, de éstas, 14 aparecen extraordinariamente deterioradas al encontrarse muy por debajo del percentil 10. En 37 casos (22 mujeres y 15 hombres), estudiamos su desarrollo comparando su talla inicial y actual en la curva de Iowa, observando que el $51,3 \%$ se mantienen en el nismo percentil, $13,5 \%$ mejoran y $35,1 \%$ disminuyen su crecimiento. De los 37 pacientes, al iniciar su diabetes, 12 están bajo el percentil 10,7 entre el 10 y 25,13 entre el 25 y 50 y sálo 5 sobre el 50 . Estos datos demuestran un deterioro previo importante del crecimiento y un mayor déficit aun durante la evolución de la diabetes.

No encontramos relación entre grado de control metabólico y crecimiento, así de 8 casos con buen control, 7 estaban bajo el percentil 10. Por Io demás, nuestros resultados son muy semejantes a los de Knowles (8) que usa dicta libre, y a los de Sterky (10) en diabéticos escandinavos, quienes encuentran este mismo déficit de crecimiento, estando en la serie de este último, más afectados los hombres que las mujeres.

Por otra parte, White, (6-11) compara curvas de peso y talla de niñas diabéticas con parientes sanas y no encuentra diferencia alguna, lo que traduce, a su modo de ver, la importancia fundamental del buen control metabólico y por lo tanto del cumplimiento de una dieta adecuada.

Respecto a menarquia, hemos observado un atraso moderado en comparación a adolescentes no diabéticas, según estadísticas nacionales (12). En nuestro grupo de enfermas, el 72\% (18 casos) presentó su menarquia entre los 12 y 15 años, en cambio en adolescentes normales hay un $82 \%$ de menarquias en ese rango de edad. Destacamos que en nuestro material hay un $12 \%$ de menarquias después de los 16 años, frente a $1,4 \%$ en normales. Estos datos coinciden con estadísticas extranjeras (8).

Nuestro promedio de menarquia (13,3 años). es totalmente comparable con los de Post (13) y Oakley.

El grado de escolaridad de este grupo de niños, no presenta diferencia significativa con lil población escolar general en este medio socioeconómico. El 53,2\% cursa enseñanza en el grado correspondiente a su edad cronológica. Estudios comparativos de diabéticos y no diabéticos en estadísticas extranjeras (6-8-14) tampoco muestran diferencias en la escolaridad de ambos grupos.

La cifrá obtenida por nosotros de Ketoacidosis diabética, $(54 \%)$ es imposible de comparar con la de autores extranjeros, debido a la absoluta disparicad de criterio en la fijación del nivel de $\mathrm{CO} 2$ que sc considera una acidosis franca (8-15). Entre las causas más frecuentes de reingreso por este motivo, deterninamos que el $68,2 \%$ se debió a suspensiones de insulina e infracciones dietéticas. En cambio en las series extranjeras se da especial importancia a infecciones, traumatismos y trastornos psicológicos (7-8).

El shock hipoglicémico que en los niños se observó en el $26,4 \%$ es similar al citado en la literatura (7-8). En los hombres fue más frecuentc $(37,9 \%)$ probablemente en relación al mayor ejercicio y a la irregularidad en el horario de las comidas, en especial en el gropo de adolescentes.

El 50\% presentó algún tipo de infección, cifra extraordinariamente alta, similar a la publicada por nosotros en adultos (16), lo cual nos permite afirmar que existe una mayor incidencia de infecciones en el diabético, en desacuerdo a lo publicado por Oakley. Stckky (17) en un análisis comparativo sobre frecuencias de infecciones agudas en escolares diabéticos y normales concluye que son más frecuentes en los diabéticos. Respecto a la localización, en dos series a las cuales nos hemos referido en otros aspectos (8-15) se observó con mayor frecuencia la infección de la piel y la urinaria, en concordancia absoluta con nuestros resultados. La tuberculosis sólo la hemos observado en 2 casos $(3 \%)$.

Existe una gran variación en las cifras respecto a la incidencia de retinopatía (18). En nuestra revisión, dentro del grupo de 41 pacientes con 6 a 19 años de diabetes, hubo 14 casos de retinopatía lo que corresponde a un $34 \%$, cifra muy dificil de comparar con otras series, ya que la duración de la diabetes es mucho mayor en observaciones extranjeras. Así, en el estudio practicado en Cincinatti (8), con un promedio de enfermedad de 18 años se obtuvo un $64 \%$ de retinopatía. En otros trabajos con observaciones de más de 30 años de diabetes, la prevalencia de retinopatía se eleva a más del $90 \%$ (19).

En una recopilación de diferentes estudios publicados en 1969 (18) se concluye que cxiste una relación estricta entre el mayor control de la diabetes y menor frecuencia de retinopatía; sin embargo, el buen control metabólico no influiría en la progresión de la retinopatía ya establecida. Nuestros resultados son concordantes, ya que $2 / 3$ de los casos con retinopatía corresponden aquellos con mal control. La incidencia de proteinuria baja y aislada en nuestra casuística (10\%), es inferior a la encontrada por Oakley $(20 \%)$. 
Sólo en dos casos pudimos formular el diagnóstica clínico de nefropatía diabética, por la presencia de albuminuria en concomitancia con retinopatía, aunque es posible que aumentara el número al realizar estudios más finos.

Los síntomas más dramáticos de neuropatía diabética se ven rara vez en niños, en nuestro grupo sólo una adolescente con pésimo control cae en esta categoría. Eeg-Olafsson (20) publica una incidencia de $1,3 \%$ de neuropatía en menores de 20 años y analiza las posibilidades de manifestaciones latentes en diabéticos infantiles asintomáticos, encontrando una disminución significativa de la velocidad de conducción nerviosa, que guarda relación con la antigiiedad de la diabetes y mal control.

\section{RESUMEN}

1.- Se estudian 63 diabéticos infantiles que iniciaron su enfermedad antes de los 15 años. 29 hombres y 34 mujeres, con un mínimo de observación de 2 años.

2.- Se destaca el hecho que $38 \%$ de ellos iniciaron la enfermedad en coma diabético y $8 \%$ en acidosis importante.

3.- Presentaron control metabólico bueno $19 \%$, regular $33 \%$ y malo $47,6 \%$ de los casos.

4.-En el grupo menor de 18 años se observó un franco retardo en el desarrollo pondoestatural, más marcado en el sexo femenino y sin relación al control metabólico. Aquellos estudiados desde la iniciación presentaron un deterioro previo importante del crecimiento $y$ un mayor déficit aun durante la evolución de la diabetes.

5.-El desarrollo normal de las niñas, medido a través de la menarquia, estaba levemente retrasado.

6.-El $54 \%$ de los casos presentó Ketoacidosis diabética en algún período de la evolución de su enjermedad.

7.-Las infecciones más frecuentes fueron de la piel y del árbol urinario, siendo éstas especialmente importantes en el grupo de mal control metabólico.

8.- El 23\% de los casos presentan Retinopatia diabética, siendo de grado leve o moderado. Existe relación entre presencia de retinopatia, antigiuedad de la diabetes y mal control metabolico.

\section{Bibliografía}

1.-Marble, A. Classification. Chapter 1I. Diabetes Mellitus: Diagnosis and Treatment, Ed. Danowski. T. S. New York, American Diabetes Association, 1964.
2.-Joslin, E. P., Root, H. F., White, F. and Marble, A. The Treatment of Diabetes Mellitus. Philadelphia. Lea and Febiger, 1959.

3.-Mella, I., García de los Ríos, M., Valiente, S., Canessa, $L, y$ Bahamondes, $G$.: Diabetes Juvenil. Bol. Hosp. S. J. de Dios 14: 56, 1967.

4.-Valiente, S. y Taucher, E.: Pcso aceptable para adultos. Bol. Hosp. S. J. de Dios 8: 15, 1961.

5.- Valiente, S., Canessa, I., Armas, R., García de los Rios, M. y Mella 1.; Acidosis diabética. Rexisión clínica. Rev. Méd. Chile 92: 430, 1964.

6.-Marble, A., White, P., Bradley, R. F. and Krall L. P. Joslin's Diabetes Mellitus. Philadelphia, Lea and Febiger, 1971.

7.-Oakley. W. G., Pyke, D. A. and Taylor, K.W. Clinical Diabetes and its Biochemical Bosis. Oxford, Blackwell scientific Publications, 1968.

8.--Knowles, M. C., Guest, G. M., Lampe, J., Kesster, M. and Skillman, T. G.: The Course of Juvenile Diabetes Treated with Unmeasured Díet. Diabetes 14: $239,1965$.

9.-Donowsky, T. S. Diabetes Mellitus with Emphasis on children and young adults. Baltimore, witliams and Wilkins, 1957.

10.-Sterky, Goratt.: Diabetic school children. Acta Paediat. (Stockholm) 52, suppl 144, 1963.

11.-White, P.: Growth and Development of Juvenilc Diabetes. $4^{\circ}$ Congres de la Fédération internationale du Diabete. Geneve, 1: 333, 1961.

12.-Jadresic, A. et al. Endocrinología, Fundumentos y Clíniça. Santiago, Ediciones de la Universidad de Chile, 1968.

13.- Post, R. R. and White, $P_{\text {.: }}$ Tentative explanation of the high incidence of diabetes. Diabetes 7: 27, 1958.

14.-Sterky, G.: Family background and state of mental bealth in a group of diabetic school children. Acta Paediat. 52: 377, 1963.

15.- White, P.: Childhood Diabetes. Its Coutce, and Influence on the second and Third generations. Diabetes 9: 345, 1960.

16.-García de los Ríos, M. Valiente, S., Cantessa. 7. Mella, L, y Tuhcher E.: Complicaciones degenerativas y sobrevida en diabéticos. Rev. Méd. Chile 100: 733,1972 .

17.-Sterky, G.: Suceptibility to infections disease, the response to active inmunization and the antibody titres in diabetic schoolchildren. Acta Paediat. 52: 252, 1963.

18.- Caird, F. I., Pirie and Ramsell, T, G. Diabetes and the Eye. Oxford, Blackweel scientific Publications. 1969.

19.-White, P.: Natural course and prognosis of Juvenile diabetics. Diabetes 5: 445, 1956.

20.-Eeg-Olofsson, C. and Petersént, I.: Childhood Diabetic Neuropatby. A Clinical and Neurophysiological study. Acta Paediat. Stockholm 55: 163, 1965. 